\title{
Synthesis of New Xanthenes Based on Lawsone and Coumarin via a Tandem Three-Component Reaction
}

\author{
Mariana F. C. Cardoso, ${ }^{\#, a}$ Luana S. M. Forezi,,$\#$ Victor G. S. Cavalcante, ${ }^{a}$ \\ Cecília S. R. Juliani, ${ }^{a}$ Jackson Antônio L. C. Resende, ${ }^{b}$ David R. da Rocha, ${ }^{a}$ \\ Fernando C. da Silva ${ }^{a}$ and Vitor F. Ferreira ${ }^{*, a}$ \\ ${ }^{a}$ Universidade Federal Fluminense, Campus do Valonguinho, 24020-150 Niterói-RJ, Brazil \\ ${ }^{b}$ Instituto de Ciências Exatas e da Terra, Universidade Federal do Mato Grosso, \\ Centro Universitário do Araguaia, 78600-000 Barra do Garças-MT, Brazil
}

\begin{abstract}
The synthesis of several new xanthenes based on the 1,3-dicarbonyl scaffolds lawsone and coumarin was developed using tandem three-component reactions involving the reagents ortho-hydroxybenzaldehyde, as bifunctional reagent, and an appropriate thiol. These reactions generated two series of para- and ortho-naphthoxanthenes that are structurally related to $\alpha$ - and $\beta$-pyranaphthoquinones and one series of coumarin-xanthene derivatives.
\end{abstract}

Keywords: 2-hydroxy-1,4-naphthoquinone, coumarin, multi-component reactions, organocatalysis, ortho-quinone methide

\section{Introduction}

Xanthenes are a class of heterocyclic compounds that are extensively found in natural products and have many biological applications and several applications in materials science. ${ }^{1-3}$ Xanthenes are prepared by several synthetic methods and structurally possess carbocyclic or heterocyclic substituents fused to a central pyran ring. In general, the most commonly used methods for their preparation involve a cycloaddition reaction of a 1,3-dicarbonyl compound with aldehydes to form an adduct, a reactive $\alpha, \beta$-unsaturated intermediate, which reacts with an appropriate nucleophile in the second step. ${ }^{4-6}$

Lawsone (1) and 4-hydroxycoumarin (6) are two 1,3-dicarbonyl compounds that have been extensively used by synthetic organic chemists because of their peculiar chemical structures and their relationships with biologically important natural products. ${ }^{7}$ Indeed, these natural products have a large variety of biological activity that has been studied in recent decades and stimulated the synthesis of a large number of synthetic analogues. ${ }^{8-11}$

Naphthoquinones play vital roles in several biological processes and are considered to be of great importance in

*e-mail: cegvito@vm.uff.br

\#These authors contributed equally to this work. medicinal chemistry. Naphthoquinones are often associated with various biological activities, including anticancer, antibacterial, antimalarial, trypanocidal, tuberculostatic and fungicidal activities. ${ }^{12}$ Some natural coumarins are found as secondary metabolites from plants, bacteria and fungi. ${ }^{13}$ This family of compounds presents a wide variety of biological activities, including anti-inflammatory, antibacterial, antifungal, antiviral and anticancer. ${ }^{14,15}$ In Figure 1, we highlight the following important natural and synthetic naphthoquinones that match this profile: lawsone (1), $\beta$-lapachone (2), $\alpha$-lapachone (3), urdamycinone B (4), estreptonigrin (5), 4-hydroxycoumarin (6), calanolide A (7) and osthole (8). ${ }^{16-19}$ Because of the importance of these naphthoquinones and coumarins, the search for new, simple and efficient methods of preparation of structurally related compounds remains of great interest.

Tandem multi-component reactions (MCR) are convergent syntheses that begin with three or more reactants and, preferably, end with only one formed product that contains all of the starting atoms. ${ }^{20}$ Usually, these types of reactions have a high atom economy and a high possibility of diversifying the structures of the final products. MCRs are important in industrial applications ${ }^{21}$ and the development of novel MCRs remains a challenge for organic chemistry because they are in accordance with green chemistry principles. In addition, they are of great interest in green 
<smiles>O=C1C=C(O)C(=O)c2ccccc21</smiles>

lawsone, 1<smiles>CC1(C)CCC2=C(O1)c1ccccc1C(=O)C2=O</smiles>

$\beta$-lapachone, 2<smiles>CC1(C)CCC2=C(O1)C(=O)c1ccccc1C2=O</smiles>

$\alpha$-lapachone, 3<smiles></smiles>

urdamycinone $\mathrm{B}, 4$<smiles>O=c1cc(O)c2ccccc2o1</smiles>

4-hydroxycoumarin, 6<smiles>CCCc1cc(=O)oc2c3c(c4c(c12)OC(C)(C)C=C4)O[C@H](C)[C@H]([14CH2][14CH3])[C@H]3O</smiles><smiles>COC1=C(N)C(=O)c2nc(-c3nc(C(=O)O)c(C)c(-c4ccc(OC)c(OC)c4O)c3N)ccc2C1=O</smiles><smiles>COc1ccc2ccc(=O)oc2c1CC=C(C)C</smiles>

osthole, 8

Figure 1. Representative of natural and synthetic bioactive naphthoquinones and coumarins.

medicinal chemistry because these reactions utilize simple methodologies and can lead to new bioactive structures. ${ }^{22-24}$

New multicomponent reactions are desired and still being discovered, proving their importance and applicability as a tool in synthetic organic chemistry. Recently, our group has developed three new multicomponent reactions to prepare naphthoquinone analogues using $o$-quinone methide intermediates (o-QMs) as the key-steps, which are formed by the reaction of $\mathbf{1}$ with various aldehydes. The first example involved the preparation of several 3-alkyl derivatives of $\mathbf{1}$, including lapachol (9) (Scheme 1), among other naphthoquinones, ${ }^{25}$ which are reduced in situ leading to 3-alkyl-1,4-naphthoquinone. The second reaction was the 1,4-addition of several thiols to the $o$-QMs. Using this method, it was possible to prepare a series of new $C$-alkyl or $C$-arylsulfanylmethyl-[1,4]-naphthoquinones (10) in good to high yields (Scheme 1). These latter compounds were screened against the human malaria parasite Plasmodium falciparum (3D7) ${ }^{26}$ and Leishmania (L.) infantum and exhibited moderate to good activity. ${ }^{27}$ The

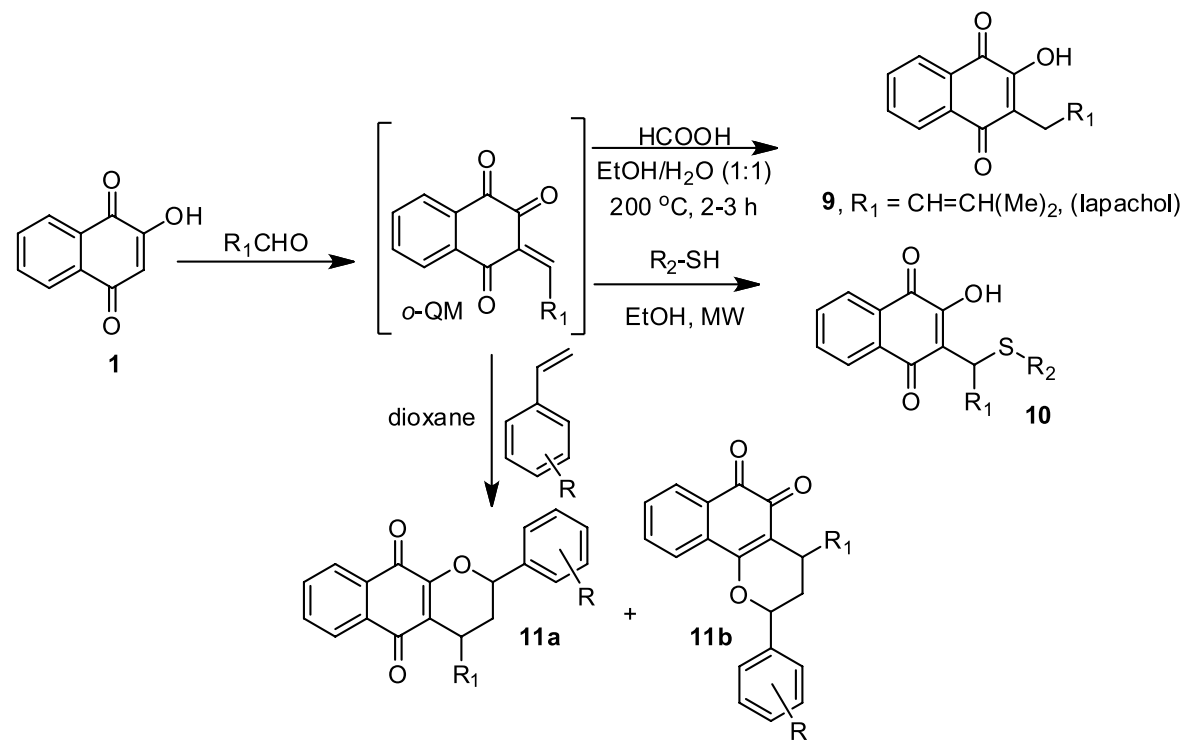

Scheme 1. New multicomponent reactions involving $o$-quinone methides. 
third process was the hetero Diels-Alder of $o$-QMs with several styrenes, forming $\alpha$ - and $\beta$-pyranaphthoquinones $11 \mathrm{a}$ and $11 \mathbf{b} .^{28}$

Xanthenes fused with ortho-quinone or para-quinone (12-16) have been reported in the literature (Figure 2). ${ }^{29-37}$ More recently, our research group became interested in the synthesis of $6 H$-dibenzo $[b, h]$ xanthenes $(\mathbf{1 6})$ that contain the two asymmetrical naphthoquinone moieties, which were prepared selectively under solvent-free conditions. ${ }^{38}$

With this aim in mind and as a further extension of our studies of the chemistry of xanthene fused with naphthoquinone and coumarin moieties, we have prepared two new series of para- and ortho-naphthoxanthenes, 17 and 18, which are structurally related to $\beta$-lapachone (2) and $\alpha$-lapachone (3), respectively, via an organocatalyzed three-component reaction using lawsone (1) as a starting material and a series of coumarinyl-xanthenes (21) from 4-hydroxycoumarin (6) (Scheme 2).

\section{Experimental}

\section{Materials and methods}

Melting points were obtained on a Thomas Hoover apparatus (Philadelphia, USA) and are uncorrected.
Analytical grade solvents were used. Column chromatography was performed using silica gel (Acros Organics 0.035-0.070 mm, pore diameter ca. $6 \mathrm{~nm}$ ). Infrared spectra were recorded on a Shimadzu IR Prestige-21 FTIR spectrometer (Kyoto, Japan). ${ }^{1} \mathrm{H}$ and ${ }^{13} \mathrm{C}$ nuclear magnetic resonance (NMR) were recorded at room temperature using a VNMRSYS-500 or a Varian MR 400 instrument, using the solvents indicated, with TMS as an internal standard. Chemical shifts $(\delta)$ are given in ppm and coupling constants $(J)$ in Hertz $(\mathrm{Hz})$. Elemental analysis was performed based on the Pregl-Dumas method and quantified using a thermal conductivity detector (PerkinElmer 2400 Series II). High-resolution mass spectra (HRMS) were recorded on a mass spectrometer MICROMASS Q-TOF (Waters, Rio de Janeiro, Brazil).

General procedures for synthesis of naphthoxanthenes 17a-g and 18a-g

In a $125 \mathrm{~mL}$ round-bottom flask containing a solution of lawsone ( $1 \mathrm{~g}, 5.74 \mathrm{mmol})$, salicylaldehyde $(0.6 \mathrm{~mL}$, $11.4 \mathrm{mmol}$ ), urea (34 mg, $0.5 \mathrm{mmol})$, and $40 \mathrm{~mL}$ of ethanol, the appropriate thiophenol $(1.2 \mathrm{mmol})$ were added. The mixture was then refluxed for $4 \mathrm{~h}$ and then the solvent was evaporated under reduced pressure. The<smiles>O=C1C2=C(C(=O)c3ccccc31)C(Br)c1c(ccc3ccccc13)O2</smiles><smiles>CC1(C)CC(=O)c2c(oc3c(c2=O)C(=O)c2ccccc2C3=O)C1</smiles><smiles></smiles><smiles></smiles><smiles></smiles>

Figure 2. Some examples of naphthoxanthenes.<smiles></smiles>

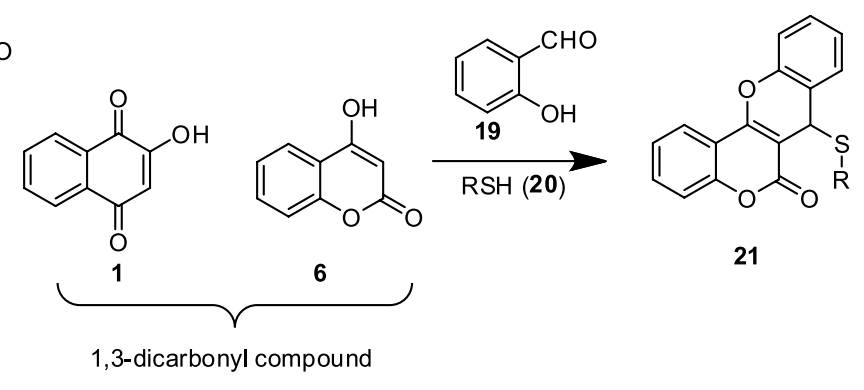

Scheme 2. The strategy used for the synthesis of xanthenes 17, 18 and $\mathbf{2 1 .}$ 
mixture was dissolved in $50 \mathrm{~mL}$ of chloroform and poured into $50 \mathrm{~mL}$ of distilled water. The phases were separated, and the organic phase was washed with saturated sodium bicarbonate solution $(3 \times 50 \mathrm{~mL})$, rinsed with distilled water $(3 \times 50 \mathrm{~mL})$, dried with anhydrous sodium sulfate, filtered and evaporated under reduced pressure. The resulting red solid was purified by flash column chromatography using silica gel and eluted with gradient mixtures of dichloromethane and ethyl acetate.

12-(Phenylthio)-11 $H$-benzo[ $b]$ xanthene-6,11(12H)-dione (17a)

Yellow solid. m.p. $166-167^{\circ} \mathrm{C}$; IR $(\mathrm{KBr}) \mathrm{v} / \mathrm{cm}^{-1} 1655$, $1628,1378,1300,1244,1216,959,721,692 ;{ }^{1} \mathrm{H}$ NMR $\left(500 \mathrm{MHz}, \mathrm{DMSO}-d_{6}\right) \delta 8.12(\mathrm{dd}, J 7.3,1.5 \mathrm{~Hz}, 1 \mathrm{H}), 8.05$ (dd, J 7.3, 1.5 Hz, 1H), 7.92 (dt, J 7.3, 1.5, Hz, 1H), 7.89 (dt, J 7.3, 1.5, Hz, 1H), 7.65-7.62 (m, 1H), 7.33-7.29 (m, 3H), $7.11(\mathrm{t}, J 7.8 \mathrm{~Hz}, 2 \mathrm{H}), 7.00-6.97(\mathrm{~m}, 1 \mathrm{H}), 6.84$ (dd, J 7.8, $0.9 \mathrm{~Hz}, 2 \mathrm{H}), 5.72(\mathrm{~s}, 1 \mathrm{H}) ;{ }^{13} \mathrm{C} \mathrm{NMR}\left(75 \mathrm{MHz}, \mathrm{DMSO}-d_{6}\right)$ $\delta 181.8,176.9,150.6,149.8,136.1,134.6,134.0,131.2$, $130.3,129.8,129.5,129.4,129.0,128.5,126.0,125.9$, 125.8, 120.0, 118.1, 116.2, 40.2. Anal. calcd. for $\mathrm{C}_{23} \mathrm{H}_{14} \mathrm{O}_{3} \mathrm{~S}$ (370.4224 $\left.\mathrm{g} \mathrm{mol}^{-1}\right)$ : C, 74.58; H, 3.81\%. Found: C, 74.56; $\mathrm{H}, 3.77 \%$.

12-((4-Chlorophenyl)thio)-11 H-benzo[b]xanthene6,11(12H)-dione (17b)

Yellow solid. m.p. $160-163{ }^{\circ} \mathrm{C}$; IR (KBr) v / $\mathrm{cm}^{-1} 1655$, 1631, 1376, 1245, 1218, 959, 720; ' $\mathrm{H}$ NMR (500 MHz, DMSO- $\left.d_{6}\right) \delta 8.12-8.08(\mathrm{~m}, 1 \mathrm{H}), 8.08-8.04(\mathrm{~m}, 1 \mathrm{H}), 7.94-7.86$ (m, 2H), 7.66-7.63 (m, 1H), 7.33-7.30 (m, 2H), 7.18 (dd, $J 8.8,2.2 \mathrm{~Hz}, 2 \mathrm{H}), 7.06-7.03(\mathrm{~m}, 1 \mathrm{H}), 6.85(\mathrm{dd}, J 8.8,2.2 \mathrm{~Hz}$, $2 \mathrm{H}), 5.75(\mathrm{~s}, 1 \mathrm{H}) ;{ }^{13} \mathrm{C}$ NMR (75 MHz, DMSO- $\left.d_{6}\right) \delta 181.7$, 176.9, 150.6, 149.9, 137.8, 134.8, 134.6, 134.0, 131.2, 130.4, 129.8, 129.2, 128.6, 128.5, 126.1, 125.9, 125.8, 119.7, 118.0, 116.3, 40.3. Anal. calcd. for $\mathrm{C}_{23} \mathrm{H}_{15} \mathrm{ClO}_{3} \mathrm{~S}\left(406.8800 \mathrm{~g} \mathrm{~mol}^{-1}\right)$ : C, 67.90; H, 3.72\%. Found: C, 67.95; H, 3.70\%.

12-((4-Tolyl)thio)-11H-benzo[b]xanthene-6,11(12H)-dione (17c)

Yellow solid. m.p. $189-190{ }^{\circ} \mathrm{C}$; IR (KBr) v / $\mathrm{cm}^{-1} 1678$, 1651, 1629, 1374, 1245, 1200, 958, 758, 716; ${ }^{1} \mathrm{H}$ NMR $\left(500 \mathrm{MHz}, \mathrm{DMSO}-d_{6}\right) \delta$ 8.11-8.09 $(\mathrm{m}, 1 \mathrm{H}), 8.06-8.03$ (m, 1H), 7.95-7.85 (m, 2H), 7.63-7.59 (m, 1H), 7.31-7.28 $(\mathrm{m}, 2 \mathrm{H}), 7.03-6.98(\mathrm{~m}, 1 \mathrm{H}), 6.91(\mathrm{~d}, J 7.9 \mathrm{~Hz}, 2 \mathrm{H}), 6.71$ $(\mathrm{d}, J 7.9 \mathrm{~Hz}, 2 \mathrm{H}), 5.65(\mathrm{~s}, 1 \mathrm{H}), 2.22(\mathrm{~s}, 3 \mathrm{H}) ;{ }^{13} \mathrm{C} \mathrm{NMR}$ $\left(75 \mathrm{MHz}, \mathrm{DMSO}-d_{6}\right) \delta 181.7,176.9,150.5,149.7,139.1$, $136.0,134.5,133.9,131.2,130.3,129.7,129.1,128.8$, 126.0, 125.9, 125.8, 125.7, 120.1, 118.2, 116.1, 40.1, 20.5. Anal. calcd. for $\mathrm{C}_{24} \mathrm{H}_{16} \mathrm{O}_{3} \mathrm{~S}$ (384.4490 $\left.\mathrm{g} \mathrm{mol}^{-1}\right)$ : C, 74.98; H, $4.20 \%$. Found: C, 75.17; H, $4.18 \%$.
12-(Cyclohexylthio)-11H-benzo[ $b]$ xanthene-6,11(12H)dione (17d)

Yellow solid. m.p. $180-182{ }^{\circ} \mathrm{C}$; IR $(\mathrm{KBr}) \mathrm{v} / \mathrm{cm}^{-1} 1684$, 1650, 1632, 1369, 1236, 959, 745, 716; ${ }^{1} \mathrm{H} \mathrm{NMR} \mathrm{(500} \mathrm{MHz,}$ DMSO- $\left.d_{6}\right) \delta 8.09-8.07(\mathrm{~m}, 2 \mathrm{H}), 7.91-7.83(\mathrm{~m}, 2 \mathrm{H}), 7.56(\mathrm{~d}$, J 7.7 Hz, 1H), 7.41-7.27 (m, 3H), 5.38 (s, 1H), 2.83-2.77 (m, 1H), 1.59-1.45 (m, 6H), 1.25-1.15 (m, 4H); ${ }^{13} \mathrm{C} \mathrm{NMR}$ $\left(75 \mathrm{MHz}, \mathrm{DMSO}-d_{6}\right) \delta 182.1,177.2,150.5,149.5,134.5$, $133.9,131.2,130.4,129.6,128.7,126.0,125.8,125.7$, 121.6, 120.7, 116.5, 43.4, 34.1, 34.0, 33.3, 25.2, 25.1, 24.9. Anal. calcd. for $\mathrm{C}_{23} \mathrm{H}_{20} \mathrm{O}_{3} \mathrm{~S}\left(376.4700 \mathrm{~g} \mathrm{~mol}^{-1}\right)$ : $\mathrm{C}, 73.38$; H, 5.36\%. Found: C, 73.40; H, 5.35\%.

12-(Benzylthio)-11H-benzo[b]xanthene-6,11(12H)-dione (17e)

Yellow solid. m.p. $247-248{ }^{\circ} \mathrm{C}$; IR (KBr) v / cm ${ }^{-1} 1678$, 1656, 1635, 1374, 1241, 1214, 958, 745, 706; ${ }^{1} \mathrm{H}$ NMR $\left(500 \mathrm{MHz}, \mathrm{DMSO}-d_{6}\right) \delta 8.10(\mathrm{dd}, J 7.5,1.1 \mathrm{~Hz}, 1 \mathrm{H}), 8.06$ (dd, J 7.4, 1.1 Hz, 1H), 7.73 (dt, J 7.4, 1.4, Hz, 1H), 7.69 (dt, J 7.4, 1.4, Hz, 1H), 7.28-7.278 (m, 3H), 7.21-7.18 (m, 3H), 7.05 (t, J 7.3 Hz, 2H), 6.96 (t, J 7.5 Hz, 1H), 5.29 (s, 1H), 3.64 (d, $J 13.5 \mathrm{~Hz}, 1 \mathrm{H}), 3.59$ (d, $J 13.5 \mathrm{~Hz}, 1 \mathrm{H}) ;{ }^{13} \mathrm{C} \mathrm{NMR}$ $\left(75 \mathrm{MHz}, \mathrm{DMSO}-d_{6}\right) \delta 182.1,177.0,150.3,149.7,137.4$, $134.2,133.7,131.2,130.4,129.8,128.9,128.3,127.9$, 126.4, 125.9, 125.8, 125.6, 120.3, 119.1, 116.4, 35.3, 33.5. Anal. calcd. for $\mathrm{C}_{24} \mathrm{H}_{16} \mathrm{O}_{3} \mathrm{~S}\left(384.4490 \mathrm{~g} \mathrm{~mol}^{-1}\right)$ : C, 74.98; H, $4.20 \%$. Found: C, 74.96; H, 4.22\%.

12-(Propylthio)-11H-benzo[b]xanthene-6,11(12H)-dione (17f)

Yellow solid. m.p. $183-184{ }^{\circ} \mathrm{C}$; IR $(\mathrm{KBr}) \mathrm{v} / \mathrm{cm}^{-1} 1682$, 1631, 1593, 1373, 1240, 958, 748, 716; ${ }^{1} \mathrm{H} \mathrm{NMR}(500 \mathrm{MHz}$, DMSO- $\left.d_{6}\right) \delta 8.09-8.04(\mathrm{~m}, 2 \mathrm{H}), 7.87-7.89(\mathrm{~m}, 2 \mathrm{H}), 7.60-7.57$ (m, 1H), 7.40-7.27 (m, 3H), $5.33(\mathrm{~s}, 1 \mathrm{H}), 2.47-2.41(\mathrm{~m}, 1 \mathrm{H})$, 2.25-2.17 (m, 1H), 1.34 (sex, J 2.3 Hz, 2H), 0.76 (t, J7.3 Hz, $2 \mathrm{H}) ;{ }^{13} \mathrm{C}$ NMR (75 MHz, DMSO- $\left.d_{6}\right) \delta 182.0,177.2,150.5$, $149.7,134.4,133.8,131.2,130.5,129.6,128.8,125.9$, 125.8, 125.7, 120.8, 119.6, 116.3, 34.6, 30.9, 21.8, 13.2. Anal. calcd. for $\mathrm{C}_{20} \mathrm{H}_{16} \mathrm{O}_{3} \mathrm{~S}\left(336.4050 \mathrm{~g} \mathrm{~mol}^{-1}\right)$ : C, 71.41; $\mathrm{H}$, 4.79\%. Found: C, 71.39; H, 4.80\%.

12-(2-Naphthalenylthio)-11H-benzo[b]xanthene-6,11(12H)dione (17g)

Yellow solid. m.p. $204-206^{\circ} \mathrm{C}$; IR (KBr) v / cm ${ }^{-1} 1675$, 1650, 1626, 1377, 1244, 1196, 957, 811, 745, 715; ${ }^{1} \mathrm{H}$ NMR $\left(500 \mathrm{MHz}, \mathrm{DMSO}-d_{6}\right) \delta 8.11(\mathrm{dd}, J 7.5,1.1 \mathrm{~Hz}, 1 \mathrm{H}), 8.01$ (dd, J 7.5, 1.2 Hz, 1H), 7.92 (dt, J 7.5, 1.2 Hz, 2H), 7.84 (d, $J 7.4 \mathrm{~Hz}, 1 \mathrm{H}), 7.65(\mathrm{dd}, J 7.4,1.1 \mathrm{~Hz}, 1 \mathrm{H}), 7.66(\mathrm{~d}, J 7.4 \mathrm{~Hz}$, 1H), 7.54-7.50 (m, 2H), 7.46 (dd, J 7.5, 1.1 Hz, 1H), 7.44 (s, 1H), 7.33-7.27 (m, 2H), 6.94 (d, J 8.4 Hz, 1H), 6.88 (d, $J 8.4 \mathrm{~Hz}, 1 \mathrm{H}), 5.79(\mathrm{~s}, 1 \mathrm{H}) ;{ }^{13} \mathrm{C}$ NMR $\left(75 \mathrm{MHz}, \mathrm{DMSO}-d_{6}\right)$ 
$\delta 181.7,176.8,150.5,149.7,136.0,134.5,133.9,132.0$, 132.6, 132.5, 131.2, 130.3, 129.7, 128.9, 127.7, 127.3, 127.2, 127.1, 126.9, 126.3, 125.9, 125.8, 125.7, 120.1, 118.3, 116.1, 40.3. Anal. calcd. for $\mathrm{C}_{27} \mathrm{H}_{16} \mathrm{O}_{3} \mathrm{~S}\left(420.4820 \mathrm{~g} \mathrm{~mol}^{-1}\right)$ : C, 77.13 ; $\mathrm{H}, 3.84 \%$. Found: C, $77.15 ; \mathrm{H}, 3.85 \%$.

\section{7-(Phenylthio)-6H-benzo[c]xanthene-5,6(7H)-dione (18a)}

Yellow solid. m.p. $172-173{ }^{\circ} \mathrm{C}$; IR $(\mathrm{KBr}) \mathrm{v} / \mathrm{cm}^{-1} 1650$, $1607,1585,1353,1263,1244,1212,1006,838,719$, $655 ;{ }^{1} \mathrm{H}$ NMR $\left(500 \mathrm{MHz}, \mathrm{DMSO}-d_{6}\right) \delta 7.96(\mathrm{~d}, J 7.6 \mathrm{~Hz}$, $1 \mathrm{H}), 7.86(\mathrm{~d}, J 7.6 \mathrm{~Hz}, 1 \mathrm{H}), 7.72-7.69(\mathrm{~m}, 1 \mathrm{H}), 7.60-7.57$ $(\mathrm{m}, 1 \mathrm{H}), 7.42-7.31(\mathrm{~m}, 4 \mathrm{H}), 7.12-7.09(\mathrm{~m}, 2 \mathrm{H}), 6.97(\mathrm{~d}$, $J 7.8 \mathrm{~Hz}, 2 \mathrm{H}), 6.93-6.90(\mathrm{~m}, 1 \mathrm{H}) 5.15(\mathrm{~s}, 1 \mathrm{H}) ;{ }^{13} \mathrm{C} \mathrm{NMR}$ $\left(75 \mathrm{MHz}, \mathrm{DMSO}-d_{6}\right) \delta 181.4,179.9,161.7,157.5,135.9$, 135.0, 133.8, 133.0, 130.9, 130.8, 129.9, 129.4, 129.3, 128.1, 126.7, 126.6, 126.0, 120.2, 118.3, 115.7, 35.1. Anal. calcd. for $\mathrm{C}_{23} \mathrm{H}_{14} \mathrm{O}_{3} \mathrm{~S}\left(370.4224 \mathrm{~g} \mathrm{~mol}^{-1}\right)$ : C, 74.58; H, 3.81\%. Found: C, 74.59; H, 3.78\%.

\section{7-(4-Chlorophenylthio)-6H-benzo[c]xanthene-5,6(7H)-dione} (18b)

Yellow solid. m.p. $168-169{ }^{\circ} \mathrm{C}$; IR $(\mathrm{KBr}) \mathrm{v} / \mathrm{cm}^{-1}$ 1655, 1636, 1588, 1540, 1346, 1242, 969, 718; ${ }^{1} \mathrm{H}$ NMR $\left(500 \mathrm{MHz}\right.$, DMSO- $\left.d_{6}\right) \delta 8.12(\mathrm{dd}, J 7.6,1.9 \mathrm{~Hz}, 1 \mathrm{H}), 8.07$ $(\mathrm{dd}, J 7.6,1.9 \mathrm{~Hz}, 1 \mathrm{H}), 8.01-8.00(\mathrm{~m}, 1 \mathrm{H}), 7.94(\mathrm{~m}, 1 \mathrm{H})$, 7.67-7.65 (m, 1H), 7.46 (dd, J 7.4, 2.1 Hz, 2H), 7.33-7.31 (m, 2H), 7.19 (d, J 7.4 Hz, 2H), 6.94 (d, J 7.4 Hz, 1H), 5.18 $(\mathrm{s}, 1 \mathrm{H}) ;{ }^{13} \mathrm{C}$ NMR $\left(75 \mathrm{MHz}, \mathrm{DMSO}-d_{6}\right) \delta 182.8,177.9$, $150.2,151.1,148.8,134.6,134.3,133.5,131.8,131.6$, $131.3,130.3,128.7,128.4,127.1,127.0,126.7,127.6$, 125.7, 125.6, 125.1, 125.0, 119.8, 118.9, 116.0, 35.6. Anal. calcd. for $\mathrm{C}_{23} \mathrm{H}_{15} \mathrm{ClO}_{3} \mathrm{~S}\left(406.8800 \mathrm{~g} \mathrm{~mol}^{-1}\right): \mathrm{C}, 67.90 ; \mathrm{H}$, $3.72 \%$. Found: C, $67.93 ; \mathrm{H}, 3.71 \%$.

\section{7-(4-Tolylthio)-6H-benzo[c]xanthene-5,6(7H)-dione (18c)}

Yellow solid. m.p. $192-193{ }^{\circ} \mathrm{C}$; IR ( $\left.\mathrm{KBr}\right) \mathrm{v} / \mathrm{cm}^{-1} 1634$, 1593, 1573, 1473, 1243, 1010, 812, 750, 719; ${ }^{1} \mathrm{H}$ NMR $\left(500 \mathrm{MHz}\right.$, DMSO- $\left.d_{6}\right) \delta 8.12(\mathrm{dd}, J 7.5,1.1 \mathrm{~Hz}, 1 \mathrm{H}), 8.06$ (dd, J 7.4, 1.2 Hz, 1H), $7.93(\mathrm{dt}, J 7.5,1.5 \mathrm{~Hz}, 1 \mathrm{H}), 7.89$ $(\mathrm{dt}, J 7.4,1.5 \mathrm{~Hz}, 1 \mathrm{H}), 7.64-7.62(\mathrm{~m}, 1 \mathrm{H}), 7.39(\mathrm{~d}, J 8.2 \mathrm{~Hz}$, 2H), 7.32-7.30 (m, 2H), $7.19(\mathrm{~d}, J 8.2 \mathrm{~Hz}, 2 \mathrm{H}), 7.02-7.00$ (m, 1H) $5.16(\mathrm{~s}, 1 \mathrm{H}), 2.22(\mathrm{~s}, 3 \mathrm{H}) ;{ }^{13} \mathrm{C}$ NMR $(75 \mathrm{MHz}$, DMSO- $\left.d_{6}\right) \delta 184.3,178.1,152.8,140.2,136.9,134.6$, 133.9, 133.8, 132.1, 130.9, 129.8, 129.6, 129.4, 129.3, 129.0, 126.9, 126.8, 126.1, 120.6, 119.2, 117.1, 41.1, 21.5. Anal. calcd. for $\mathrm{C}_{24} \mathrm{H}_{16} \mathrm{O}_{3} \mathrm{~S}\left(384.4490 \mathrm{~g} \mathrm{~mol}^{-1}\right)$ : C, 74.98 ; H, $4.20 \%$. Found: C, $75.02 ; \mathrm{H}, 4.21 \%$.

7-(Cyclohexylthio)-6H-benzo[c]xanthene-5,6(7H)-dione (18d)

Yellow solid. m.p. $160-163{ }^{\circ} \mathrm{C}$; IR $(\mathrm{KBr}) v / \mathrm{cm}^{-1} 1651$,
$1589,1538,1369,1342,1270,1242,1220,939,757,717$; ${ }^{1} \mathrm{H}$ NMR $\left(500 \mathrm{MHz}\right.$, DMSO- $\left.d_{6}\right) \delta 8.16(\mathrm{~d}, J 7.6 \mathrm{~Hz}, 1 \mathrm{H})$, 8.09 (d, J 7.6 Hz, 1H), 7.69 (dd, J 7.6, 1.3 Hz, 1H), 7.63$7.61(\mathrm{~m}, 1 \mathrm{H}), 7.50(\mathrm{dt}, J 7.9,1.2 \mathrm{~Hz}, 1 \mathrm{H}), 7.25-7.13(\mathrm{~m}$, $3 \mathrm{H}), 5.22(\mathrm{~s}, 1 \mathrm{H}), 4.05-4.01(\mathrm{~m}, 1 \mathrm{H}), 1.59-1.54(\mathrm{~m}, 2 \mathrm{H})$, $1.33-1.07$ (m, 8H); ${ }^{13} \mathrm{C}$ NMR (75 MHz, DMSO- $\left.d_{6}\right) \delta 187.4$, 181.7, 178.2, 151.7, 148.8, 134.8, 134.7, 134.6, 133.9, 132.7, 130.1, 129.4, 128.7, 127.4, 126.7, 123.7, 118.9, 48.9, 43.4, 33.3, 32.2, 25.4, 25.2, 25.1. Anal. calcd. for $\mathrm{C}_{23} \mathrm{H}_{20} \mathrm{O}_{3} \mathrm{~S}\left(376.4700 \mathrm{~g} \mathrm{~mol}^{-1}\right)$ : C, 73.38; H, 5.36\%. Found: C, 73.37 ; H, 5.33\%.

7-(Benzylthio)-6H-benzo[c]xanthene-5,6(7H)-dione (18e)

Red solid. m.p. 233-235 ${ }^{\circ} \mathrm{C}$; IR (KBr) v / $\mathrm{cm}^{-1} 1634$, 1593, 1573, 1243, 1010, 958, 812, 750, 719; ${ }^{1} \mathrm{H}$ NMR $\left(500 \mathrm{MHz}, \mathrm{DMSO}-d_{6}\right) \delta 8.04(\mathrm{dd}, J 7.9,1.2 \mathrm{~Hz}, 1 \mathrm{H}), 7.91$ (dd, J 7.9, 1.2 Hz, 1H), 7.68 (dt, J 7.9, $1.2 \mathrm{~Hz}, 1 \mathrm{H}), 7.54$ (dt, J 7.9, 1.2 Hz, 1H), 7.30-7.27 (m, 1H), 7.24-7.22 (m, $3 \mathrm{H}), 7.21-7.18(\mathrm{~m}, 2 \mathrm{H}), 7.06(\mathrm{t}, J 7.7 \mathrm{~Hz}, 2 \mathrm{H}), 6.93(\mathrm{~m}, 1 \mathrm{H})$, 5.17 (s, $1 \mathrm{H}), 3.69$ (q, J $14.5 \mathrm{~Hz}, 2 \mathrm{H}) ;{ }^{13} \mathrm{C}$ NMR $(75 \mathrm{MHz}$, DMSO- $\left.d_{6}\right) \delta 182.2,177.1,150.4,149.8,137.4,134.3$, $133.8,131.3,130.5,129.9,129.0,126.5,128.4,128.0$, 126.0, 125.9, 125.9, 125.7, 120.3, 119.1, 116.4, 35.3, 33.4. Anal. calcd. for $\mathrm{C}_{24} \mathrm{H}_{16} \mathrm{O}_{3} \mathrm{~S}\left(384.4490 \mathrm{~g} \mathrm{~mol}^{-1}\right)$ : C, 74.98; H, 4.20\%. Found: C, 74.99; H, 4.21\%.

\section{7-(Propylthio)-6H-benzo[c]xanthene-5,6(7H)-dione (18f)}

Yellow solid. m.p. 203-206 ${ }^{\circ} \mathrm{C}$; IR (KBr) v / cm ${ }^{-1} 1643$, 1588, 1536, 1347, 1244, 1221, 970, 744, 720; ${ }^{1} \mathrm{H}$ NMR $\left(500 \mathrm{MHz}\right.$, DMSO- $\left.d_{6}\right) \delta 8.12(\mathrm{dd}, J 7.3,1.3 \mathrm{~Hz}, 1 \mathrm{H}), 8.07$ (dd, $J 7.3,1.3 \mathrm{~Hz}, 1 \mathrm{H}), 7.94(\mathrm{dd}, J 7.2,1.3 \mathrm{~Hz}, 1 \mathrm{H}), 7.91(\mathrm{dd}, J 7.3$, $1.3 \mathrm{~Hz}, 1 \mathrm{H}), 7.67-7.65(\mathrm{~m}, 1 \mathrm{H}), 7.37-7.31(\mathrm{~m}, 3 \mathrm{H}), 5.04(\mathrm{~s}$, $1 \mathrm{H}), 2.05-1.97$ (m, 2H), 1.20-1.08 (m, 2H), 0.55 (t, J 7.3 Hz, $3 \mathrm{H}) ;{ }^{13} \mathrm{C}$ NMR (75 MHz, DMSO- $\left.d_{6}\right) \delta 183.4,181.8,177.1$, 153.0, 150.5, 137.8, 134.8, 134.7, 134.0, 133.1, 132.5, 129.4, 126.2, 126.0, 125.9, 119.8, 119.7, 118.1, 116.3, 38.3, 31.7, 22.7, 14.9. Anal. calcd. for $\mathrm{C}_{20} \mathrm{H}_{16} \mathrm{O}_{3} \mathrm{~S}\left(336.4050 \mathrm{~g} \mathrm{~mol}^{-1}\right)$ : C, $71.41 ; \mathrm{H}, 4.79 \%$. Found: C, 71.40; H, 4.77\%.

\section{7-(2-Naphthalenylthio)-6H-benzo[c]xanthene-5,6(7H)-dione (18g)}

Yellow solid. m.p. $184-185^{\circ} \mathrm{C}$; IR (KBr) v / cm ${ }^{-1} 1654$, 1587, 1533, 1362, 1268, 1005, 810, 725, 681; ${ }^{1} \mathrm{H}$ NMR $\left(500 \mathrm{MHz}\right.$, DMSO- $\left.d_{6}\right) \delta 8.15(\mathrm{dd}, J 7.5,1.0 \mathrm{~Hz}, 1 \mathrm{H}), 8.08$ $(\mathrm{dd}, J 7.4,1.0 \mathrm{~Hz}, 1 \mathrm{H}), 7.89(\mathrm{~m}, 1 \mathrm{H}), 7.82(\mathrm{~m}, 1 \mathrm{H}), 7.70-7.59$ (m, 6H), 7.32-7.21 (m, 3H), 7.02-6.94 (m, 2H), $5.13(\mathrm{~s}, 1 \mathrm{H})$; ${ }^{13} \mathrm{C} \mathrm{NMR}\left(75 \mathrm{MHz}\right.$, DMSO- $\left.d_{6}\right) \delta 180.5,180.2,162.7,143.9$, 139.5, 134.4, 132.8, 133.2, 133.0, 130.9, 130.5, 127.9, 127.4, 126.7, 126.3, 126.1, 126.0, 125.9, 125.2, 124.8, 121.1, 118.6, 113.8, 38.3. Anal. calcd. for $\mathrm{C}_{27} \mathrm{H}_{16} \mathrm{O}_{3} \mathrm{~S}\left(420.4820 \mathrm{~g} \mathrm{~mol}^{-1}\right)$ : C, $77.13 ; \mathrm{H}, 3.84 \%$. Found: C, 77.12; H, 3.85\%. 
General procedures for synthesis of coumarinyl-xanthenes 21a-j

In a $125 \mathrm{~mL}$ round-bottom flask containing a solution of 4-hydroxy-coumarin ( $1 \mathrm{~g}, 6.2 \mathrm{mmol})$, salicylaldehyde $(12.3 \mathrm{mmol})$ and $20 \mathrm{~mL}$ of ethanol, the appropriate thiophenol $(12.3 \mathrm{mmol})$ were added. The mixture was then refluxed for $48 \mathrm{~h}$. The obtained solid was filtered and washed with iced petroleum ether. Then, the product was purified by recrystallization in ethanol.

7-(Phenylthio)-6H,7H-chromeno[4,3-b]chromen-6-one (21a)

White solid. m.p. $188-190{ }^{\circ} \mathrm{C}$; IR (KBr) v / cm ${ }^{-1} 1718$, 1633, 1573, 1393, 1243, 1044, 972, 872, 748, 697, 665; ${ }^{1} \mathrm{H}$ NMR $\left(500 \mathrm{MHz}, \mathrm{DMSO}-d_{6}\right) \delta 7.72-7.67(\mathrm{~m}, 2 \mathrm{H}), 7.60$ (dd, J 6.9, $2.3 \mathrm{~Hz}, 1 \mathrm{H}), 7.49$ (d, J 8.2 Hz, 1H), 7.42-7.38 (m, 1H), 7.35-7.29 (m, 2H), 7.29-7.24 (m, 1H), 7.29-7.22 (m, 1H), 7.09-6.99 (m, 3H), 6.79 (d, J 1.2 Hz, 1H), 5.55 $(\mathrm{s}, 1 \mathrm{H}) ;{ }^{13} \mathrm{C} \mathrm{NMR} / \mathrm{attached}$ proton test (APT) $(75 \mathrm{MHz}$, DMSO- $\left.d_{6}\right) \delta 159.6,156.1,151.9,149.6,136.2,132.8$, $129.9,129.4,129.0,128.5,125.9,124.7,122.4,116.6$, 116.1, 100.2, 41.8. Calcd. for $\mathrm{C}_{22} \mathrm{H}_{14} \mathrm{NaO}_{3} \mathrm{~S}^{+}$: 381.0556 . Found for $\mathrm{C}_{22} \mathrm{H}_{14} \mathrm{NaO}_{3} \mathrm{~S}^{+}: 381.0560$.

7-(p-Tolylthio)-6H,7H-chromeno[4,3-b]chromen-6-one (21b)

White solid. m.p. 179-181 ${ }^{\circ} \mathrm{C}$; IR $(\mathrm{KBr}) \mathrm{v} / \mathrm{cm}^{-1}$ 1721, 1642, 1488, 1394, 1221, 761; ${ }^{1} \mathrm{H}$ NMR (500 MHz, DMSO- $\left.d_{6}\right) \delta 7.75(\mathrm{dd}, J 7.9,1.4 \mathrm{~Hz}, 1 \mathrm{H}), 7.73-7.68(\mathrm{~m}$, 1H), 7.59 (dd, J 7.3, 2.0 Hz, 1H), 7.50 (dd, J 8.3, 0.6 Hz, $1 \mathrm{H}), 7.43-7.40$ (m, 1H), 7.32 (dpent, J 7.3, $1.7 \mathrm{~Hz}, 2 \mathrm{H}$ ), 7.10 (dd, J 7.7, $1.7 \mathrm{~Hz}, 1 \mathrm{H}), 6.86$ (d, J 7.8 Hz, 2H), 6.68 $(\mathrm{d}, J 8.0 \mathrm{~Hz}, 2 \mathrm{H}), 5.50(\mathrm{~s}, 1 \mathrm{H}), 2.18(\mathrm{~s}, 3 \mathrm{H}) ;{ }^{13} \mathrm{C} \mathrm{NMR} / \mathrm{APT}$ $\left(75 \mathrm{MHz}, \mathrm{DMSO}-d_{6}\right) \delta 159.3,155.9,151.7,149.4,138.9$, $135.9,132.6,129.6,128.9,128.7,125.6,124.4,122.2$, 116.4, 115.9, 100.2, 41.6, 20.5. Calcd. for $\mathrm{C}_{23} \mathrm{H}_{16} \mathrm{NaO}_{3} \mathrm{~S}^{+}$: 395.0712. Found for $\mathrm{C}_{23} \mathrm{H}_{16} \mathrm{NaO}_{3} \mathrm{~S}^{+}: 395.0720$.

7-((4-Chlorophenyl)thio)-6H,7H-chromeno[4,3- $b]$ chromen6-one (21c)

White solid. m.p. $180-182{ }^{\circ} \mathrm{C}$; IR $(\mathrm{KBr}) \mathrm{v} / \mathrm{cm}^{1}$ 1719, 1639, 1485, 1396, 1221, 761; ${ }^{1} \mathrm{H}$ NMR (300 MHz, DMSO- $\left.d_{6}\right) \delta 7.76(\mathrm{dd}, J 7.9,1.5 \mathrm{~Hz}, 1 \mathrm{H}), 7.73-7.65(\mathrm{~m}$, $1 \mathrm{H}), 7.61-7.53(\mathrm{~m}, 1 \mathrm{H}), 7.48(\mathrm{dd}, J 8.3,0.8 \mathrm{~Hz}, 1 \mathrm{H})$, 7.45-7.38 (m, 1H), 7.38-7.26 (m, 2H), 7.15-7.08 (m, 1H), $7.12(\mathrm{~d}, J 8.5 \mathrm{~Hz}, 2 \mathrm{H}), 6.83(\mathrm{~d}, J 8.5 \mathrm{~Hz}, 2 \mathrm{H}), 5.57$ (s, $1 \mathrm{H}) ;{ }^{13} \mathrm{C}$ NMR/APT $\left(75 \mathrm{MHz}\right.$, DMSO- $\left.d_{6}\right) \delta 159.3,156.0$, $151.8,149.4,137.5,132.7,129.7,129.0,128.3,125.8$, $124.5,122.2,120.5,116.5,116.0,100.1,41.9$. Calcd. for $\mathrm{C}_{22} \mathrm{H}_{13} \mathrm{ClNaO}_{3} \mathrm{~S}^{+}$: 415.0166. Found for $\mathrm{C}_{22} \mathrm{H}_{13} \mathrm{ClNaO}_{3} \mathrm{~S}^{+}$: 415.0172.
7-((4-Methoxyphenyl)thio)-6H,7H-chromeno[4,3-b] chromen-6-one (21d)

White solid. m.p. $152-153{ }^{\circ} \mathrm{C}$; IR (KBr) v / $\mathrm{cm}^{-1} 1717$, 1637, 1587, 1483, 1455, 1394, 1286, 1246, 1173, 1033, 841, 761, 670; ${ }^{1} \mathrm{H}$ NMR (500 MHz, DMSO- $\left.d_{6}\right) \delta 7.74$ (dd, J 7.9, 1.4 Hz, 1H), 7.75-7.68 (m, 1H), 7.61-7.59 (m, 1H), $7.50(\mathrm{dd}, J 8.3,0.5 \mathrm{~Hz}, 1 \mathrm{H}), 7.43-7.41$ (dt, $J$ 7.9, $1.0 \mathrm{~Hz}, 1 \mathrm{H}), 7.34-7.31(\mathrm{~m}, 2 \mathrm{H}), 7.10-7.08(\mathrm{~m}, 1 \mathrm{H}), 6.66(\mathrm{~d}$, $J 8.9 \mathrm{~Hz}, 2 \mathrm{H}), 6.58(\mathrm{~d}, J 8.8 \mathrm{~Hz}, 2 \mathrm{H}), 5.45(\mathrm{~s}, 1 \mathrm{H}), 3.63(\mathrm{~s}$, $3 \mathrm{H}) ;{ }^{13} \mathrm{C}$ NMR/APT $\left(75 \mathrm{MHz}, \mathrm{DMSO}-d_{6}\right) \delta 160.4,159.4$, $155.9,151.8,149.5,137.8,132.6,129.8,128.8,125.7$, $124.5,122.2,120.9,120.1,116.5,115.9,113.9,113.2$, 100.2, 55.1, 41.7. Calcd. for $\mathrm{C}_{23} \mathrm{H}_{16} \mathrm{NaO}_{4} \mathrm{~S}^{+}$: 411.0662 . Found for $\mathrm{C}_{23} \mathrm{H}_{16} \mathrm{NaO}_{4} \mathrm{~S}^{+}: 411.0673$.

7-(Cyclohexylthio)-6H,7H-chromeno[4,3-b]chromen-6-one (21e)

White solid. m.p. $124-125^{\circ} \mathrm{C}$; IR (KBr) v / $\mathrm{cm}^{-1} 2926$, 2845, 1712, 1638, 1483, 1450, 1383, 1330, 1273, 1240, 1222, 1173, 1104, 1047, 900, 749; 'H NMR (500 MHz, DMSO- $\left.d_{6}\right) \delta 8.06(\mathrm{dd}, J 7.8,1.3 \mathrm{~Hz}, 1 \mathrm{H}), 7.75-7.71(\mathrm{~m}$, 1H), $7.56(\mathrm{~d}, J 7.7 \mathrm{~Hz}, 1 \mathrm{H}), 7.51-7.47$ (m, 2H), 7.41 (d, $J 7.8 \mathrm{~Hz}, 2 \mathrm{H}), 7.34-7.30(\mathrm{~m}, 1 \mathrm{H}), 5.27$ (s, 1H), 2.85-2.78 (m, 1H), 1.84-1.79 (m, 1H), 1.68-1.66 (m, 1H), 1.61-1.55 (m, 3H), $1.45(\mathrm{~m}, 1 \mathrm{H}), 1.22-1.11(\mathrm{~m}, 4 \mathrm{H}) ;{ }^{13} \mathrm{C}$ NMR/APT $\left(75 \mathrm{MHz}, \mathrm{DMSO}-d_{6}\right) \delta 159.6,155.5,151.7,149.3,132.6$, $129.6,128.6,125.7,124.5,122.3,122.2,116.4,116.3$, 113.4, 102.5, 43.2, 35.5, 34.1, 33.3, 25.2, 24.9. Calcd. for $\mathrm{C}_{22} \mathrm{H}_{20} \mathrm{KO}_{3} \mathrm{~S}^{+}:$403.0765. Found for $\mathrm{C}_{22} \mathrm{H}_{20} \mathrm{KO}_{3} \mathrm{~S}^{+}: 403.0769$.

7-((4-Fluorophenyl)thio)-6H,7H-chromeno[4,3-b]chromen6-one (21f)

White solid. m.p. 206-207 ${ }^{\circ} \mathrm{C}$; IR (KBr) v / $\mathrm{cm}^{-1} 1716$, 1638, 1495, 1397, 1221, 1046, 755; ${ }^{1} \mathrm{H}$ NMR (500 MHz, DMSO- $\left.d_{6}\right) \delta 7.76(\mathrm{dd}, J 7.9,1.4 \mathrm{~Hz}, 1 \mathrm{H}), 7.71$ (ddd, $J$ 8.8, 7.3, 1.6 Hz, 1H), 7.63 (dd, J 7.0, $2.4 \mathrm{~Hz}, 1 \mathrm{H}), 7.51$ (dd, J 8.3, 0.5 Hz, 1H), 7.44-7.41 (m, 1H), 7.36-7.31 (m, 2H), 7.11 (dd, J 7.7, 1.7 Hz, 1H), 6.89 (t, J 8.9 Hz, 2H), $6.80(\mathrm{dd}, J 8.8,5.6 \mathrm{~Hz}, 2 \mathrm{H}), 5.56(\mathrm{~s}, 1 \mathrm{H}) ;{ }^{13} \mathrm{C} \mathrm{NMR} / \mathrm{APT}$ $\left(75 \mathrm{MHz}, \mathrm{DMSO}-d_{6}\right) \delta 162.9\left(\mathrm{~d},{ }^{1} J_{\mathrm{C}-\mathrm{F}}=247.8 \mathrm{~Hz}\right), 159.3$, $155.9,151.8,149.5,138.4\left(\mathrm{~d},{ }^{3} J_{\mathrm{C}-\mathrm{F}}=8.8 \mathrm{~Hz}\right), 129.7,128.9$, $125.7,125.4\left(\mathrm{~d},{ }^{4} J_{\mathrm{C}-\mathrm{F}}=3.2 \mathrm{~Hz}\right), 124.5,122.2,120.5,116.5$, $115.9,115.4\left(\mathrm{~d},{ }^{2} J_{\mathrm{C}-\mathrm{F}}=21.8 \mathrm{~Hz}\right), 113.1,100.1,41.8$. Calcd. for $\mathrm{C}_{22} \mathrm{H}_{13} \mathrm{FNaO}_{3} \mathrm{~S}^{+}$: 399.0462 . Found for $\mathrm{C}_{22} \mathrm{H}_{13} \mathrm{FNaO}_{3} \mathrm{~S}^{+}$: 399.0480 .

7-((4-Hydroxyphenyl)thio)-6H,7H-chromeno[4,3-b] chromen-6-one (21g)

White solid. m.p. $223-224{ }^{\circ} \mathrm{C}$; IR (KBr) v / $\mathrm{cm}^{-1} 3234$, 1689, 1638, 1494, 1486, 1402, 1271, 1221, 1065, 758; ${ }^{1} \mathrm{H}$ NMR (500 MHz, DMSO- $\left.d_{6}\right) \delta 9.60$ (s, 1H), 7.74 (dd, 
$J 7.9,1.4 \mathrm{~Hz}, 1 \mathrm{H}), 7.70$ (ddd, $J 8.8,7.4,1.6 \mathrm{~Hz}, 1 \mathrm{H}), 7.59$ $(\mathrm{dd}, J 6.9,2.4 \mathrm{~Hz}, 1 \mathrm{H}), 7.50(\mathrm{dd}, J 8.3,0.4 \mathrm{~Hz}, 1 \mathrm{H}), 7.44-7.38$ $(\mathrm{m}, 1 \mathrm{H}), 7.32(\mathrm{~m}, 2 \mathrm{H}), 7.09-7.07(\mathrm{~m}, 1 \mathrm{H}), 6.52(\mathrm{~d}, J 8.6 \mathrm{~Hz}$, $2 \mathrm{H}), 6.39(\mathrm{~d}, J 8.6 \mathrm{~Hz}, 2 \mathrm{H}), 5.39(\mathrm{~s}, 1 \mathrm{H}) ;{ }^{13} \mathrm{C}$ NMR/APT $\left(75 \mathrm{MHz}\right.$, DMSO- $\left.d_{6}\right) \delta 159.4,158.6,155.7,151.7,149.5$, $137.8,132.4,129.7,128.6,125.5,124.3,122.1,116.4,115.8$, 115.3, 100.2, 41.6. Calcd. for $\mathrm{C}_{23} \mathrm{H}_{14} \mathrm{NaO}_{4} \mathrm{~S}^{+}$: 397.0505 . Found for $\mathrm{C}_{23} \mathrm{H}_{14} \mathrm{NaO}_{4} \mathrm{~S}^{+}: 397.0513$.

\section{7-(Naphthalen-2-ylthio)-6H,7H-chromeno[4,3-b]chromen-} 6-one (21h)

White solid. m.p. $175-177{ }^{\circ} \mathrm{C}$; IR (KBr) v / $\mathrm{cm}^{-1}$ $1716,1638,1484,1397,1242,1220,1045,758 ;{ }^{1} \mathrm{H}$ NMR $\left(500 \mathrm{MHz}, \mathrm{DMSO}-d_{6}\right) \delta 7.81(\mathrm{~d}, J 8.0 \mathrm{~Hz}, 1 \mathrm{H}), 7.72-7.67$ $(\mathrm{m}, 1 \mathrm{H}), 7.66-7.62(\mathrm{~m}, 1 \mathrm{H}), 7.59(\mathrm{~d}, J 8.5 \mathrm{~Hz}, 1 \mathrm{H}), 7.56-$ $7.52(\mathrm{~m}, 1 \mathrm{H}), 7.51(\mathrm{~d}, J 8.5 \mathrm{~Hz}, 1 \mathrm{H}), 7.55-7.49(\mathrm{~m}, 1 \mathrm{H})$, $7.46(\mathrm{~d}, J 7.5 \mathrm{~Hz}, 1 \mathrm{H}), 7.43-7.38(\mathrm{~m}, 1 \mathrm{H}), 7.39(\mathrm{~s}, 1 \mathrm{H}), 7.36-$ $7.29(\mathrm{~m}, 4 \mathrm{H}), 6.99-6.97(\mathrm{~m}, 1 \mathrm{H}), 6.90(\mathrm{dd}, J 8.4,1.8 \mathrm{~Hz}$, $1 \mathrm{H}), 5.67(\mathrm{~s}, 1 \mathrm{H}) ;{ }^{13} \mathrm{C}$ NMR/APT $\left(75 \mathrm{MHz}\right.$, DMSO- $\left.d_{6}\right)$ $\delta$ 159.5, 156.0, 151.8, 149.4, 132.1, 129.2, 127.7, 127.6, 127.3, 127.2, 126.9, 126.8, 126.3, 126.2, 115.9, 100.2, 41.9. Calcd. for $\mathrm{C}_{26} \mathrm{H}_{16} \mathrm{NaO}_{3} \mathrm{~S}^{+}$: 431.0712. Found for $\mathrm{C}_{26} \mathrm{H}_{16} \mathrm{NaO}_{3} \mathrm{~S}^{+}$: 431.0723 .

\section{7-((4-Nitrophenyl)thio)-6H,7H-chromeno[4,3-b]chromen-} 6-one (21i)

White solid. m.p. $210-211^{\circ} \mathrm{C}$; IR $(\mathrm{KBr}) v / \mathrm{cm}^{-1} 1715$, 1639, 1573, 1501, 1457, 1393, 1342, 1239, 1221, 853, 764, 759; ${ }^{1} \mathrm{H}$ NMR (500 MHz, DMSO- $\left.d_{6}\right) \delta 8.24$ (d, $J 9.0 \mathrm{~Hz}$, $1 \mathrm{H}), 7.97(\mathrm{~d}, J 8.9 \mathrm{~Hz}, 2 \mathrm{H}), 7.87$ (dd, $J 7.9,1.5 \mathrm{~Hz}, 1 \mathrm{H})$, 7.80 (d, $J 9.0 \mathrm{~Hz}, 1 \mathrm{H}), 7.76-7.72$ (ddd, $J 8.8,7.4,1.6 \mathrm{~Hz}$, $1 \mathrm{H}), 7.60(\mathrm{dd}, J 7.7,1.6 \mathrm{~Hz}, 1 \mathrm{H}), 7.47-7.45(\mathrm{dt}, J$ 7.5, $1.6 \mathrm{~Hz}, 1 \mathrm{H}), 7.40-7.37$ (dt, J 7.5, $1.6 \mathrm{~Hz}, 1 \mathrm{H}), 7.32$ (m, $1 \mathrm{H}), 7.32(\mathrm{~d}, J 8.9 \mathrm{~Hz}, 2 \mathrm{H}), 5.87(\mathrm{~s}, 1 \mathrm{H}) ;{ }^{13} \mathrm{C} \mathrm{NMR} / \mathrm{APT}$ $\left(75 \mathrm{MHz}\right.$, DMSO- $\left.d_{6}\right) \delta 159.4,156.3,151.9,149.3,147.2$, $140.5,134.9,132.9,129.5,129.3,126.8,125.9,124.6$, 124.3, 123.1, 122.4, 120.5, 113.1, 100.1, 41.9. Calcd. for $\mathrm{C}_{22} \mathrm{H}_{13} \mathrm{NNaO}_{5} \mathrm{~S}^{+}$: 426.0407. Found for $\mathrm{C}_{22} \mathrm{H}_{13} \mathrm{NNaO}_{5} \mathrm{~S}^{+}$: 426.0393.

7-((4-(Methylthio)phenyl)thio)-6H,7H-chromeno[4,3-b] chromen-6-one (21j)

White solid. m.p. 193-194 ${ }^{\circ} \mathrm{C}$; IR (KBr) $v / \mathrm{cm}^{-1} 1717$, 1638, 1486, 1392, 1220, 1048, 754; ${ }^{1} \mathrm{H}$ NMR (500 MHz, DMSO- $\left.d_{6}\right) \delta 7.76(\mathrm{dd}, J 7.9,1.4 \mathrm{~Hz}, 1 \mathrm{H}), 7.71(\mathrm{dt}, J 7.5$, $1.6 \mathrm{~Hz}, 1 \mathrm{H}), 7.60(\mathrm{dd}, J 7.4,1.7 \mathrm{~Hz}, 1 \mathrm{H}), 7.50(\mathrm{~d}, J 8.3 \mathrm{~Hz}$, $1 \mathrm{H}), 7.42(\mathrm{t}, J 7.6 \mathrm{~Hz}, 1 \mathrm{H}), 7.37-7.30(\mathrm{~m}, 2 \mathrm{H}), 7.13(\mathrm{dd}$, $J 7.8,1.3 \mathrm{~Hz}, 1 \mathrm{H}), 6.92(\mathrm{~d}, J 8.4 \mathrm{~Hz}, 2 \mathrm{H}), 6.71(\mathrm{~d}, J 8.3 \mathrm{~Hz}$, 2H), $5.53(\mathrm{~s}, 1 \mathrm{H}), 2.35$ (s, 3H); ${ }^{13} \mathrm{C}$ NMR/APT $(75 \mathrm{MHz}$, DMSO- $\left.d_{6}\right) \delta 159.3,155.9,151.8,149.4,140.4,136.3$, 132.6, 129.7, 128.8, 125.7, 125.6, 125.5, 124.4, 122.2,
120.9, 113.2, 100.2, 41.8, 14.3. Calcd. for $\mathrm{C}_{23} \mathrm{H}_{16} \mathrm{NaO}_{3} \mathrm{~S}_{2}{ }^{+}$: 427.0433. Found for $\mathrm{C}_{23} \mathrm{H}_{16} \mathrm{NaO}_{3} \mathrm{~S}_{2}^{+}: 427.0413$.

\section{Results and Discussion}

In this work we focused on the synthesis, via a cyclocondensation reaction, of two new families of xanthenes employing two 1,3-dicarbonyl compounds as follows: lawsone (1) and 4-hydroxycoumarin (6), orthohydroxybenzaldehyde (19) and an appropriate thiol (20). These reactions involved the following tandem reaction: in situ generation of $o$-QM through a Knoevenagel condensation, a thiol addition and a cyclocondensation.

With compound $\mathbf{1}$, two isomeric families of para- and ortho-naphthoxantenes were obtained, labeled $\mathbf{1 7}$ and 18, respectively. Initially, the reaction was attempted in ethanol under conventional heating and microwave irradiation. Both conditions led to a complex mixture of compounds. However, when it was performed using urea as an organocatalyst, the reaction proceeded smoothly and supposedly formed the appropriate intermediate, which underwent nucleophilic addition by the thiols. Finally, the species underwent thermal cyclization leading to para-naphthoxanthene derivatives 17a-g and orthonaphthoxanthenes 18a-g as minor products (entries 1-14 in Table 1). In both series of compounds we observed some lower yields for aliphatic thiols. This can be explained due to the fact that the intermediate $o$-quinone methide is a soft electrophile and has greater affinity with aromatic thiol type nucleophiles. Interestingly, when diphenyl urea was employed as a catalyst, the selectivity of some reactions inverted (see entries 1-6), and compounds 18a-c,g were obtained as the major products (Scheme 3, Table 1).

In all reactions involving urea, the paranaphthoxanthenes, 17a-g, were the major products in an approximate ratio of 2:1. These results are consistent with those previously described in the literature, ${ }^{39-43}$ which indicate that the para-isomer always forms in greater proportion due to the greater reactivity of the leading isomer of $o$-quinone methide. In reactions using diphenyl urea, the ratio is reversed $(1: 2)$ for $\mathrm{R}=\mathrm{Ph}$, indicating that the steric effects around the $o$-quinone methide intermediate are driving the selectivity of the reactions.

The isomers were separated by column chromatography using silica gel and eluent mixtures consisting of a dichloromethane/ethyl acetate gradient. All isomers were fully characterized by spectroscopic methods and highresolution mass spectra (see Supplementary Information).

Regarding the organocatalysis, ureas are able to act as catalysts in asymmetric reactions mainly through hydrogen bonds with neutral functions present in the 


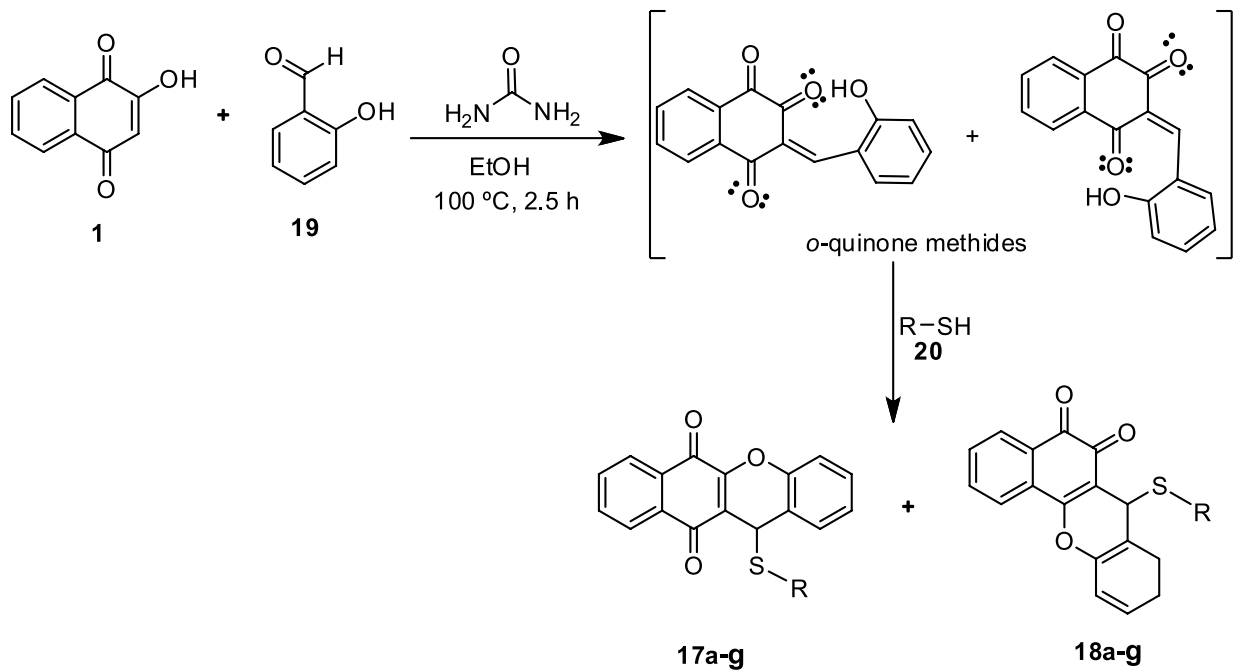

Scheme 3. Multicomponent reaction for preparing para- and ortho-naphthoxanthenes.

Table 1. Melting point and yields of the naphthoxanthenes 17a-g and 18a-g

\begin{tabular}{|c|c|c|c|c|c|}
\hline entry & Compound & $\mathrm{R}$ & $\begin{array}{c}\text { Yield }^{\mathrm{a}} / \\
\%\end{array}$ & $\begin{array}{c}\text { Yield }^{\mathrm{b}} / \\
\%\end{array}$ & $\begin{array}{c}\text { m.p. / } \\
{ }^{\circ} \mathrm{C}\end{array}$ \\
\hline 1 & $17 \mathbf{a}$ & & 60 & 31 & $166-167$ \\
\hline 2 & $18 \mathbf{a}$ & & 35 & 63 & $172-173$ \\
\hline 3 & $17 b$ & & 50 & 28 & $160-163$ \\
\hline 4 & $18 b$ & & 36 & 65 & $168-169$ \\
\hline 5 & $17 \mathrm{c}$ & & 51 & 30 & $189-190$ \\
\hline 6 & $18 \mathrm{c}$ & & 36 & 63 & $192-193$ \\
\hline 7 & 17d & & 58 & 56 & $247-248$ \\
\hline 8 & $18 d$ & & 37 & 35 & $160-163$ \\
\hline 9 & $17 \mathrm{e}$ & & 56 & 64 & $247-248$ \\
\hline 10 & $18 \mathrm{e}$ & & 30 & 28 & 233-235 \\
\hline 11 & $17 f$ & & 63 & 60 & 183-184 \\
\hline 12 & $18 f$ & & 20 & 31 & 203-206 \\
\hline 13 & $17 \mathrm{~g}$ & & 57 & 33 & 184-185 \\
\hline 14 & $18 \mathrm{~g}$ & & 31 & 58 & 204-206 \\
\hline
\end{tabular}

${ }^{a}$ Urea as catalyst; ${ }^{b}$ diphenylurea as catalyst.

substrates. ${ }^{44-46} \mathrm{~A}$ variety of important reactions, especially those involving additions to $\mathrm{C}=\mathrm{O}$ or $\mathrm{C}=\mathrm{N}$, which are accompanied by a change in the basicity of the heteroatom, are susceptible to asymmetric catalysis using derivatives of urea. Therefore, enantioselective reactions promoted by these types of organocatalysts are basically directed through non-covalent interactions, particularly hydrogen bonds, differing from those in which the catalysts depend on covalent interactions with substrates to products for inducing asymmetry. ${ }^{47}$

In this context, it was hypothesized that hydrogen bonding of the urea may occur with one (Scheme 4, Eq. 2) or two carbonyls (Scheme 4, Eq. 1) of the o-quinone methide intermediate $(o-\mathrm{QM})$ intermediate leading to its activation toward a quick addition of the thiols followed by a cyclization step (Scheme 4). Thus, the coordination of the urea with two carbonyls of the $o$-QM leads to the preferential formation of para-naphthoxanthene isomers 17a-g. When coordination occurs in only one carbonyl, the ortho-naphthoxanthenes isomers 18a-g are formed preferentially (Scheme 4). Although the aforementioned reaction with urea as a catalyst reacted quite successfully, in sharp contrast, diphenyl urea inverted the selectivity when $\mathrm{R}=\mathrm{Ph}$ (entries 1-6). It seems that the more crowded catalyst favored the formation of $\mathbf{1 8 a}-\mathbf{c}$ since the phenyl group has limited spatial conformations in the intermediate complexed with one carbonyl.

The structure of compound $\mathbf{1 7} \mathbf{a}$ was confirmed by single crystal X-ray diffraction experiments (Figure 3) following a well established method.

The analysis of compound 17a was performed on a Bruker D8 Venture diffractometer at LARE-DRX (Universidade Federal Fluminense, Brazil). The structure was solved by direct methods and refined by full-matrix least squares on $\mathrm{F}^{2}$ using the SHELX-2013 package, and all non-hydrogen atoms were refined with anisotropic displacement parameters. Hydrogen atoms bonded to $\mathrm{C}$ atoms were placed at their idealized positions using standard geometric criteria. ${ }^{48}$ The $\pi-\pi$ stacking interactions in 17a guide the conformation of the molecule and the crystal packing. The intramolecular interaction between heterocyclic and thiophenol rings shows the centroid- 


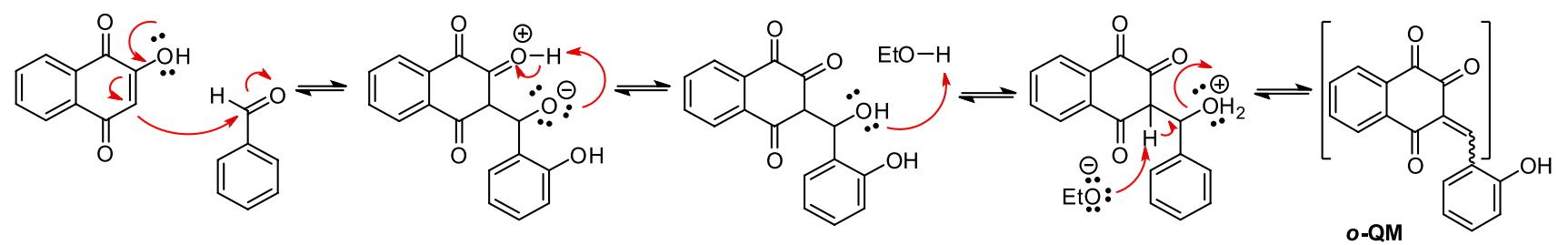

para-naphthoxanthenes $17 \mathbf{a}-\mathbf{g}$

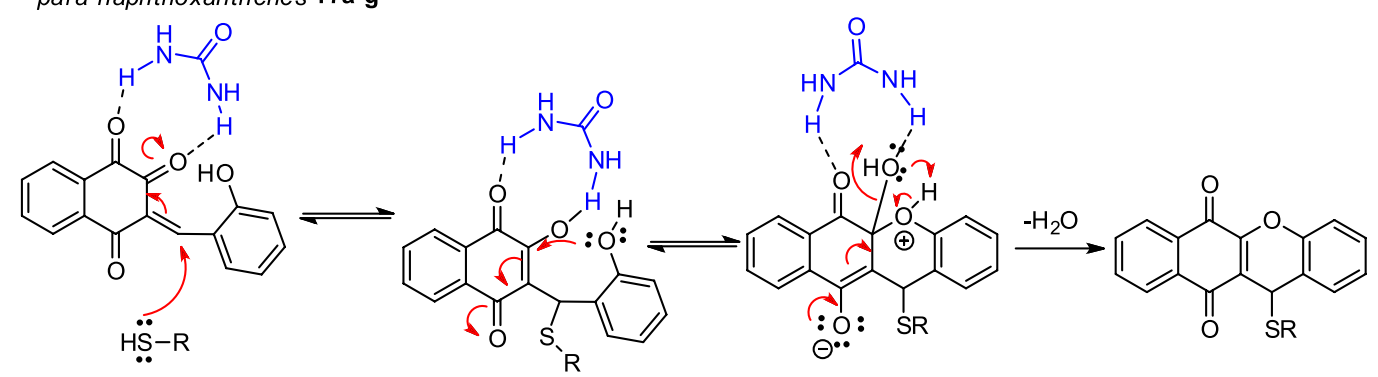

Intermediate

(Eq. 1)

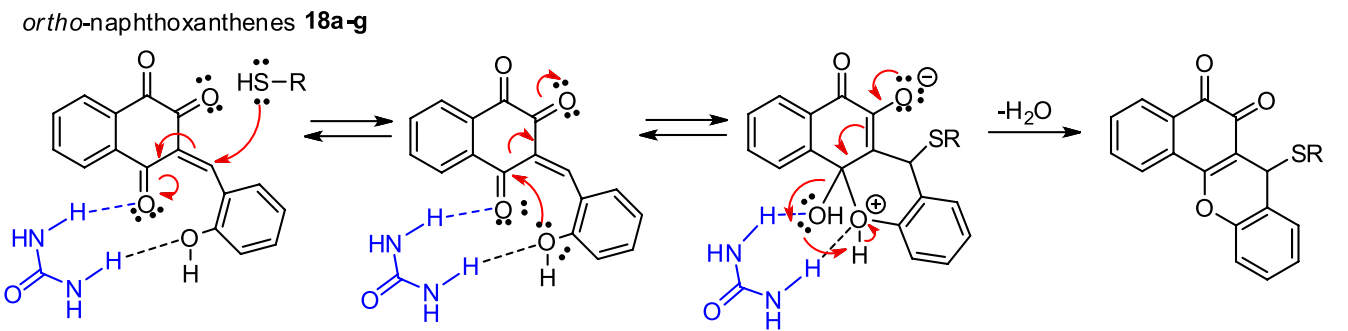

(Eq. 2)

Scheme 4. Proposed mechanism for formation of the isomers 17a-g and 18a-g.

centroid distance is 3.560(2) $\AA$. This contact possibly stabilizes the conformation of the molecule. The crystal packing presents intermolecular $\pi-\pi$ stacking between the quinone ring and the thiophenol of the adjacent molecule, which results in a chain along the direction (Figure 3). The centroid-centroid $\pi$-stacking distance is 3.525(2) $\AA$, which is typical of aromatic-aromatic face-to-face $\pi-\pi$ stacking. These crystallographic characteristics are similar to other naphthoquinones reported in the literature. ${ }^{41}$ Cambridge Crystallographic Data Centre has assigned the structure the following deposition number: CCDC 1473232 (Supplementary Information).

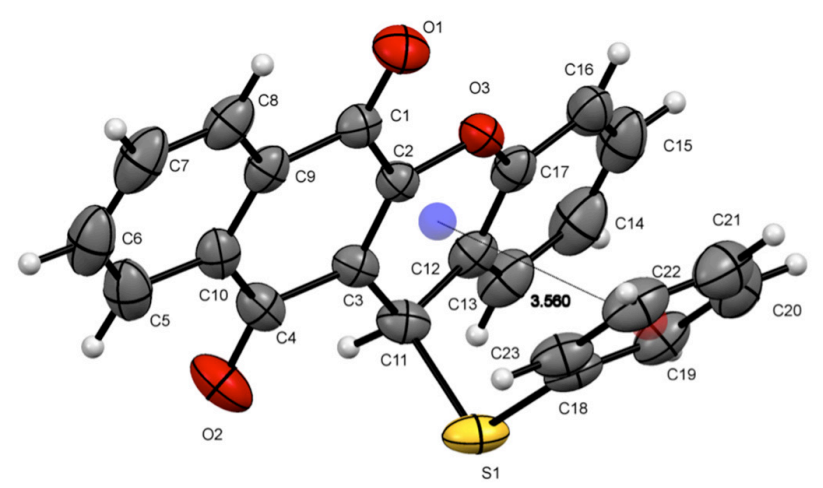

Figure 3. ORTEP representation (30\% probability displacement ellipsoid) of 1,4-naphthoxanthene 17a. Hydrogen, carbon, nitrogen, and oxygen atoms are represented in white, gray, blue, and red, respectively.
Continuing our interest in the chemistry of tandem multicomponent reactions, we decided to prepare a second family of xanthenes employing the same procedure using 4-hydroxy-coumarin (6) as the 1,3-dicarbonyl compound. The reaction was easily performed in ethanol under refluxing to obtain coumarinyl-xanthenes compounds 21a-j in good yields (Table 2). Because of the high reactivity of compound 6 toward formation of the $o-\mathrm{QM}$ intermediate it was not necessary to use an organocatalyst. Only one product was obtained in these reactions, and they were fully characterized by spectroscopic methods and high resolution mass spectra as described in the Supplementary Information.

In this case, only one isomer has been formed since the carbonyl of the ketone is more reactive than the lactone carbonyl. Thus, the nucleophilic addition of the thiophenol, the $\mathrm{C}=\mathrm{C}$ bond of the quinone methide promoted the attack of the phenolic hydroxyl to the ketone carbonyl according to the mechanism of Scheme 5.

\section{Conclusions}

The multicomponent reactions are convergent, and most of the atoms are transferred to the product in tandem, with multiple connections formed in a sequence, without isolating intermediates, changes in reaction conditions 


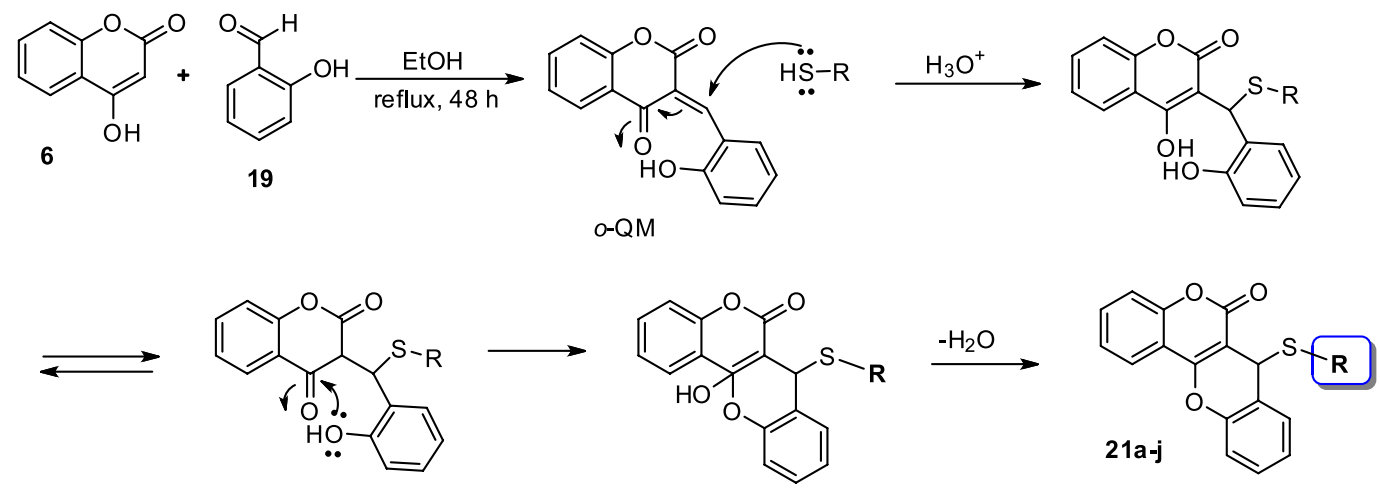

Scheme 5. Tandem multicomponent reaction for preparing the coumarinyl-xanthenes $\mathbf{2 1 a - j . ~}$

or addition of reagents. Because this process is not an equilibrium, reactions lead directly to the main product, do not produce by-products and can be used to construct a library of compounds with a high degree of complexity. The

Table 2. Melting point and yields of the coumarinyl-xanthenes 21a-j

211

two processes described above fit with these concepts. Two new series of heterocyclic derivatives of xanthenes were prepared in high yield without the isolation of intermediates and are structurally related to two 1,3-dicarbonylic natural products. Additionally, these series increased the molecular diversity of lawsone and 4-hydroxycoumarin and are suitable candidates for application in both combinatorial chemistry and drug discovery.

\section{Supplementary Information}

Supplementary data are available free of charge at http://jbcs.sbq.org.br as PDF file.

Crystallographic data (excluding structure factors) for the structures in this work were deposited in the Cambridge Crystallographic Data Centre as supplementary publication number CCDC 1473232. Copies of the data can be obtained, free of charge, via www.ccdc.cam.ac.uk/conts/retrieving. html or from the Cambridge Crystallographic Data Centre, CCDC, 12 Union Road, Cambridge CB2 1EZ, UK; fax: +44 1223 336033. E-mail: deposit@ccdc.cam.ac.uk.

\section{Acknowledgments}

The authors would like to acknowledge the agencies that fund our research: CNPq (National Council of Research of Brazil), CAPES, FAPERJ and FIOCRUZ (for the HRMS). Fellowships granted by CNPq, CAPES and FAPERJ are gratefully acknowledged. This work was partially supported by Capes grant number 058862/2010 and CNPq-FAPERJPRONEX grant number E-26/110.574/2010.

\section{References}

1. Moosophon, P.; Kanokmedhakul, S.; Kanokmedhakul, K.; Soytong, K.; J. Nat. Prod. 2009, 72, 1442.

2. Hay, A. E.; Aumond, M. C.; Mallet, S.; Dumontet, V.; Litaudon, M.; Rondeau, D.; Richomme, P.; J. Nat. Prod. 2004, 67, 707. 
3. Hashim, N.; Rahmani, M.; Sukari, M. A.; Ali, A. M.; Alitheen, N. B.; Go, R.; Ismail, H. B. M.; J. Asian Nat. Prod. Res. 2010, $12,106$.

4. Yaragorla, S.; Saini, P. L.; Babu, P. V.; Almansour, A. I.; Arumugam, N.; Tetrahedron Lett. 2016, 57, 2351.

5. Huang, H.; Yao, Y.; Lin, Q.; Zhao, J.; Hua, C.; Gou, X.; Russ. J. Gen. Chem. 2016, 86, 934.

6. Tabatabaeian, K.; Zanjanchi, M. A.; Mamaghani, M.; Dadashi, A.; Res. Chem. Intermed. 2016, 42, 5049.

7. Rodrigues, T.; Reker, D.; Schneider, P.; Schneider, G.; Nature 2016, 8, 531 .

8. Jordão, A. K.; Vargas, M. D.; Pinto, A. C.; da Silva, F. C.; Ferreira, V. F.; RSC Adv. 2015, 5, 67909.

9. Priyanka; Sharma, R. K.; Katiyar, D.; Synthesis 2016, 48, 2303.

10. Gaudino, E. C.; Tagliapietra, S.; Martina, K.; Palmisano, G.; Cravotto, G.; RSC Adv. 2016, 6, 46394.

11. Govori, S.; Synth. Commun. 2016, 46, 569.

12. da Silva, F. C.; Ferreira, V. F.; Curr. Org. Synth. 2016, 13, 334.

13. Iranshahi, M.; Askari, M.; Sahebkar, A.; Hadjipavlou-Litina, D.; DARU 2009, 17, 99.

14. Venugopala, K. N.; Rashmi, V.; Odhav, B.; BioMed Res. Int. 2013, 2013, ID963248.

15. Thakur, A.; Singla, R.; Jaitak, V.; Eur. J. Med. Chem. 2015, 101, 476.

16. Bringmann, G.; Reichert, Y.; Kane, V. V.; Tetrahedron 2004, 60, 3539.

17. Hussain, H.; Krohn, K.; Ahmad, V. U.; Miana, G. A.; Green, I. R.; Arkivoc 2007, 145.

18. Fedoryshyn, M.; Nur-E-Alam, M.; Zhu, L.; Luzhetskyy, A.; Rohr, J.; Bechthold, A.; J. Biotechnol. 2007, 130, 32.

19. Salas, C.; Tapia, R. A.; Ciudad, K.; Armstrong, V.; Orellana, M.; Kemmerling, U.; Ferreira, J.; Maya, J. D.; Morello, A.; Bioorg. Med. Chem. 2008, 16, 668.

20. Parsons, P. J.; Penkett, C. S.; Shell, A. J.; Chem. Rev. 1996, 96, 195.

21. Müller, T. J. J.; Beilstein J. Org. Chem. 2014, 10, 115.

22. Biggs-Houck, J. E.; Younai, A.; Shaw, J. T.; Curr. Opin. Chem. Biol. 2010, 14, 371.

23. Shaabani, A.; Maleki, A.; Rezayan, A. H.; Sarvary, A.; Mol. Diversity 2011, 15, 41.

24. Batalha, P. N.; Rev. Virtual Quim. 2012, 4, 13.

25. Ferreira, S. B.; da Rocha, D. R.; Carneiro, J. W. M.; Santos, W. C.; Ferreira, V. F.; Synlett 2011, 1551.

26. Sharma, A.; Santos, I. O.; Gaur, P.; Ferreira, V. F.; Garcia, C. R. S.; da Rocha, D. R.; Eur. J. Med. Chem. 2013, 59, 48.

27. Pinto, E. G.; Santos, I. O.; Schmidt, T. J.; Borborema, S. E. T.; Ferreira, V. F.; Rocha, D. R.; Tempone, A. G.; PLoS One 2014, 9, e105127.
28. da Rocha, D. R.; de Souza, A. C. G.; Resende, J. A. L. C.; Santos, W. C.; dos Santos, E. A.; Pessoa, C.; de Moraes, M. O.; Costa-Lotufo, L. V.; Montenegro, R. C.; Ferreira, V. F.; Org. Biomol. Chem. 2011, 9, 4315.

29. Rahmati, A.; Chin. Chem. Lett. 2010, 21, 761.

30. Tavakoli, H. R.; Moosavi, S. M.; Bazgir, A.; Res. Chem. Intermed. 2015, 41, 3041.

31. Chen, Y.; Wu, S.; Tu, S.; Li, C.; Shi, F.; J. Heterocycl. Chem. 2008, 45, 931.

32. Shaterian, H. R.; Rigi, F.; Res. Chem. Intermed. 2015, 41, 721.

33. Mohammadpour, A. M.; Yousef, F.; Zahra, Y.; Shahzad, F.; Ayoob, B.; Open Catal. J. 2009, 2, 40.

34. Khaligh, N. G.; Catal. Sci. Technol. 2012, 2, 2211.

35. Liu, D.; Gao, J.; Li, L.; Zhou, S.; Xu, D.; Chem. Heterocycl. Compd. 2013, 49, 1370.

36. Shaterian, H. R.; Sedghipour, M.; Mollashahi, E.; Res. Chem. Intermed. 2014, 40, 1345.

37. Bazgir, A.; Tisseh, Z. N.; Mirzaei, P.; Tetrahedron Lett. 2008, 49, 5165.

38. Carneiro, P. F.; Pinto, M. C. F. R.; Marra, R. K. F.; Campos, V. R.; Resende, J. A. L. C.; Delarmelina, M.; Carneiro, J. W. M.; Lima, E. S.; da Silva, F. C.; Ferreira, V. F.; J. Org. Chem. 2016, $81,5525$.

39. Ferreira, V. F.; Coutada, L. C.; Pinto, M. C. F. R.; Pinto, A. V.; Synth. Commun. 1982, 12, 195.

40. Ferreira, S. B.; da Silva, F. C.; Pinto, A. C.; Gonzaga, D. T. G.; Ferreira, V. F.; J. Heterocycl. Chem. 2009, 46, 1080.

41. Nair, V.; Treesa, P. M.; Maliakal, D.; Rath, N. P.; Tetrahedron 2001, 57, 7705 .

42. da Silva, F. C.; Ferreira, S. B.; Kaiser, C. R.; Pinto, A. C.; Ferreira, V. F.; J. Braz. Chem. Soc. 2009, 20, 1478.

43. da Rocha, D. R.; de Souza, A. C. G.; Resende, J. A. L. C.; Santos, W. C.; dos Santos, E. A.; Pessoa, C.; de Moraes, M. O.; Costa-Lotufo, L. V.; Montenegro, R. C.; Ferreira, V. F.; Org. Biomol. Chem. 2011, 9, 4315.

44. Wang, C. J.; Dong, X. Q.; Zhang, Z. H.; Xue, Z. Y.; Teng, H. L.; J. Am. Chem. Soc. 2008, 130, 8606.

45. Sonsona, I. G.; Marqués-López, E.; Herrera, R. P.; Beilstein J. Org. Chem. 2016, 12, 505.

46. Odagi, M.; Yamamoto, Y.; Nagasawa, K.; Beilstein J. Org. Chem. 2016, 12, 198.

47. Valero, G.; Schimer, J.; Cisarova, I.; Vesely, J.; Moyano, A.; Rios, R.; Tetrahedron Lett. 2009, 50, 1943.

48. Sheldrick, G. M.; Acta Crystallogr., Sect. A 2008, 64, 112.

Submitted: November 21, 2016

Published online: February 16, 2017 